\title{
ANTECEDENTES BASICOS SOBRE EL COMPORTAMIENTO ESTRUCTURAL \\ DE DIAFRAGMAS VERTICALES SOLICITADOS POR CARGAS HORIZONTALES INDUCIDAS EN SU PLANO
}

Víctor A. Carvallo A. ( $\left.{ }^{*}\right)$

\begin{abstract}
RESUMEN
Este artículo, entrega algunos antecedentes básicos del comportamiento estructural de panales revestidos con placas y sometidos a carga horizontal en un plano. Se entrega la teoría fundamental para poder determinar la relación cargadeformación en muros de corte y se señala la metología experimental, actualmente en vigencia. La investigación ha demostrado que el mayor aporte en el desplazamiento horizontal de un panel viene dado por el corrimiento de las fijaciones (sobre el $80 \%$ ), por lo que es primordial su correcta evaluación. Este trabajo entrega cuatro tipos de ecuaciones que representan la curva cargadeformación de las fijaciones: exponencial, logarítmica, asintótica y tangente hiperbólica. La mejor predicción es entregada por la curva asintótica.

Por otra parte la experiencia ha demostrado que la carga admisible recomendada para este tipo de panel, es aquella que induce una deformación de $\mathrm{H} / 300$, siendo $\mathrm{H}$ la altura del panel $(8 \mathrm{~mm}$. en muro de $240 \mathrm{~cm}$. de alto).
\end{abstract}

\section{ABSTRACT}

This article gives some basic information on the structural behaviour of plate coated panels under horizontal loads. General theory and up-dated methodology to determine load-strain ratios in shear walls are discused.

Research has demonstrated that the main contribution to horizontal displacement is caused by the sliding of fasteners (over $80 \%$ ), therefore its correct evaluation is essential.

This work presents four kinds of equations representing the load-strain curve for fasteners, namely: exponential, logarithmic, asymtotic, and hyperbolical tangent.

On the other hand, ethis work has shown that recommended allowable loads for this kind of panel are those that induce a maximun strain of $H / 300$, being $H$ the height of the panel $(8 \mathrm{~mm}$. on a wall of $240 \mathrm{~cm}$. high). 


\section{INTRODUCCION}

No obstante los importantes avances obtenidos en el conocimiento del comportamiento estructural de la madera, tanto en Chile como en el extranjero, uno de los aspectos en los cuales todavía no existe una concepción uniforme a nivel mundial, es el relacionado con la caracterización analítica de diafragmas rigidizados con placas, las que facilitan la construcción, permiten la industrialización y con los cuales se obtienen capacidades resistentes mayores respecto de los muros tradicionales rigidizados con diagonales o entablados.

Un diafragma es un elemento estructural delgado, normalmente rectangular, formado fundamentalmente por un bastidor sobre el cual se fija una placa o revestimiento, capaz de soportar solicitaciones de cizalle y que por rigidez limita las deformaciones de una estructura.

Considerando los tipos de solicitaciones a que quedan expuestos los diafragmas y su disposición dentro de la estructura, es conveniente dividirlos en:

\section{a) Diafragmas Horizontales}

Son elementos planos horizontales o inclinados que corresponden a pisos o techos de edificios que, además de resistir solicitaciones verticales de peso propio y sobrecarga, pueden soportar fuerzas de viento e inercia y transmitirlas a las subestructuras verticales conectadas a ellos.

\section{b) Diafragmas Verticales}

Son elementos planos verticales resistentes a la acción de fuerzas laterales, y tienen la función de servir de apoyo a los diafragmas horizontales y transferir las cargas laterales a las fundaciones.

Los diafragmas verticales paralelos a la carga lateral aplicada, reciben el nombre de muros de corte.

Los muros de corte juegan un rol importante en la resistencia a las solicitaciones de viento y sismo en construcciones que utilizan diafragmas de madera, obteniéndose a través de ellos una rigidización de toda la estructura.

La importancia de conocer su comportamiento, ha llevado a investigadores extranjeros a presentar diferentes modelos que tratan de determinar la relación carga-deformación de los paneles y sus resistencia máxima.

De todas estas investigaciones, se ha logrado concluir que el comportamiento de un muro de madera sometido a carga horizontal, es esencialmente gobernado por las características de. las fijaciones (comúnmente clavos o tornillos), de las cuales se sabe, cumplen una relación, carga-deformación, altamente no lineal. El aporte rigidizante debido a la flexión en los pies derechos y al corte en el revestimiento, tienen una influencia secundaria. 


\section{FIGURA 1}

\section{COMPONENTES TIPICOS DE UN MURO REVESTIDO CON PLACAS}

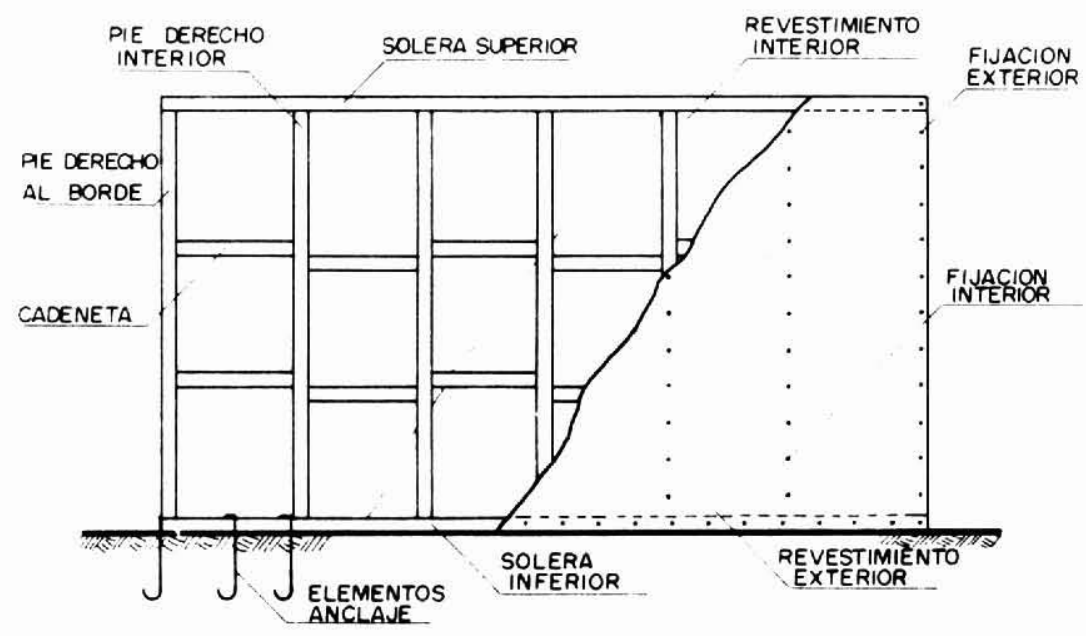

\section{TEORIA GENERAL}

Los diafragmas rigidizados por un entablado diagonal corresponden, al igual que los diafragmas rigidizados con una o dos diagonales, a sistemas constructivos que se utilizaron masivamente antes de la incorporación al mercado de los tableros de madera reconstituida (contrachapados, fibra, partículas) u otro material (fibro-cemento, cartón-yeso). La desventaja de los diafragmas tradicionales con respecto a los rigidizados por placas, radica en que el primero presenta un comportamiento distinto dependiendo del sentido de la carga solicitante. Además de ser más lentos de construir, exigen un mayor volumen de materia prima para alcanzar una capacidad resistente similar a los diafragmas rigidizados con placas.

Es por esto, que en los países industrializados se ha preferido el uso de tableros como elementos rigidizantes para los muros de madera.

Las solicitaciones a las cuales está afecto un muro son:

\section{- Cargas Verticales} él.

Que son aquellas de peso propio y sobrecarga de las estructuras que están soportadas por 


\section{- Cargas Horizontales}

Provenientes de la acción del viento y sismo. Estas cargas pueden solicitar al muro en forma perpendicular o paralela según la dirección longitudinal de éste.

Una modelación para comprender el comportamiento estructural de un diafragma vertical de madera, es asimilarlo a una viga de acero del tipo I que trabaja en voladizo. En clla, los esfuerzos axiales de tracción y compresión son absorbidos a través de los pies derechos (alas de la viga), y el esfucrzo de corte es absorbido por la placa rigidizante (alma de la viga).

\section{FICURA 2}

\section{ANALO(IIA ENTRE MURO REVESTIDO Y VIGA DE ACERO}

$\mathbf{P}$

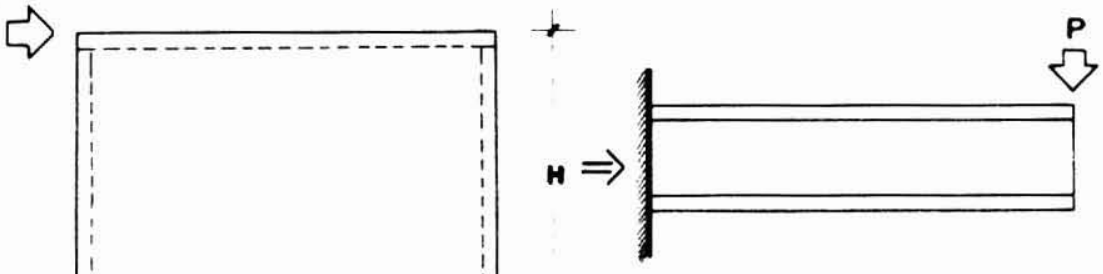

Lo esencial para el buen comportamiento estructural de un diafragma es lograr una respuesta ductil de éste, sin perdida excesiva de resistencia ante una cierta solicitación. Debido a que la madera falla en forma frágil, la ductibilidad de los muros ha de asegurarse mediante un apropiado diseño de las fijaciones (número, tipo y proporción) que unen la placa yel marco, y de los elementos de anclaje. Con esto se pretende que la falla en el muro se origine al ceder las uniones y no en los otros materiales constitutivos.

La carga horizontal que solicita a una estructura se distribuye entre líneas resistentes de acuerdo a la rigidez relativa entre los diafragmas verticales y los horizontales. La solución exacta se encuentra entre dos situaciones extremas:

a) Elementos verticales infinitamente rígidos unidos por un diafragma horizontal flexible, ó

b) Elementos verticales flexibles unidos por un diafragma horizontal infinitamente rígido.

El primer caso corresponde al método de las áreas tributarias que ha sido aplicado 
tradicionalmente en estructuras de madera, y el segundo corresponde a la hipótesis que se asume en edificios de hormigón armado, en donde esta situación, también se puede resolver en forma simplificada, asimilando el problema al de una viga apoyada sobre resortes, en donde la rigidez de ella representa al diafragma horizontal, y las rigideces de los resortes representan a los muros paralelos a la carga solicitante.

En todo caso, la distribución de las cargas laterales para los elementos resistentes, considerada como proporción del área tributaria, sólo debe ser usada en estructuras secundarias o en diafragmas apoyados en sus extremos.

Independiente del modelo que se aplique para analizar una estructura, resulta necesario conocer la rigidez de los diafragmas, para lo cual es imprescindible determinar la curva cargadeformación de éstos.

Debido a la acción de una carga lateral sobre un diafragma vertical y de la interacción de todas sus partes, se concluye que la deformación horizontal en su plano se puede descomponer en:

- Deformación de la fijación placa-bastidor.

- Deformación por corte de la placa.

- Deformación de los pies derechos y soleras.

- Deformación de los elementos de anclaje.

Se puede demostrar que la mayor contribución proviene de la deformación de las fijaciones, las cuales tienen un comportamiento no lineal y que, en definitiva, ellas son las principales responsables de la conducta dúctil que tiene un muro de madera

La rigidez a la flexión de los pies derechos, la rigidez al corte del revestimiento y la rigidez de los elementos de anclaje juegan un rol secundario en la definición de las propiedades de carga-deformación de un muro.

En lo que respecta al fenómeno de pandeo en el revestimiento, el problema de diseño no ha sido totalmente establecido. Sin embargo, es improbable que ocurra en tableros con espesores superiores a $9 \mathrm{~mm}$.

\section{METODO EXPERIMENTAL}

Los modelos que se puedan proponer para predecir el comportamiento estructural de paneles sometidos a carga horizontal son esencialmente teóricos y, por lo tanto, deben ser confrontados con los resultados de ensayos reales con el fin de determinar su grado de exactitud. 802 E Of. 70.

La forma de proceder para la experiment ación está establecida en la norma chilena $\mathrm{NCH}$

A continuación se entrega un resumen de los principales aspectos contemplados en ella:

\section{Alcance}

La norma establece el método para comprobar la resistencia de los paneles prefabricados a las cargas horizontales contenidas en su plano. Ella es aplicable a pancles prefabricados destinados a constituir elementos de una edificación, ya sea que estén formados por un sólo material o por diversos materiales unidos para trabajar en conjunto. 


\section{Resumen del Método}

Consiste en someter un panel elegido como muestra a la acción de cargas que se incrementan gradualmente y medir las deformaciones producidas por cada incremento hasta la fluencia del material. Luego sin medir deformaciones, llevar las cargas hasta la ruptura del panel.

Basándose en las lecturas efectuadas durante el ensayo, se podrá dibujar un gráfico que establezca la relación carga-deformación. Este gráfico permitirá determinar, entre otras cosas la carga de trabajo admisible y el respectivo coeficiente del elemento estudiado. Igualmente, se podrá determinar el límite de fluencia y detectar otros fenómenos que se produzcan durante el ensayo, tales como alabeos, daños locales o ruptura.

\section{Aparatos y Accesorios}

Se montarán como se indica en la Figura № 3 
FIGURA 3

PLANTA, ELEVACION Y CORTE DE LA MAQUINA Y APARATOS DE ENSAYO
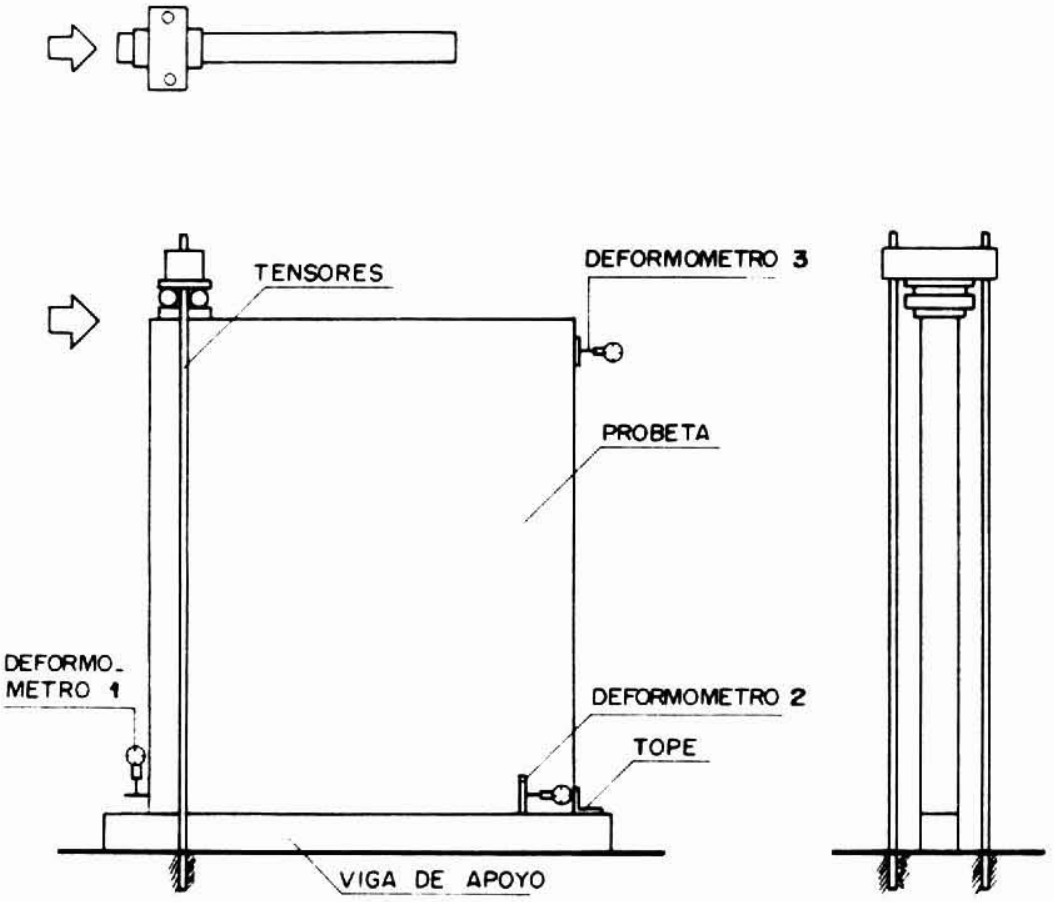

En la Figura № 3, el deformómetro 1 mide cualquier levantamiento del pancl. El deformómetro 2 registra cualquier desplazamiento del panel. El deformómetro 3 mide cl total de los otros dos desplazamientos más la deformación del panel.

El sistema de anclaje lo constituyen tensores anclados a la base de apoyo, placas repartidoras de cargas y rodillos de acero, que se montan según se indica en la Figura №3. Estc sistema debe restringir al mínimo posible todo el levantamiento de la base del panel, para no dificultar la deformación longitudinal de éste.

Debe proveerse al mecanismo de un bastidor o elemento rígido que, dotado de rodillos 
u otro sistema similar, impida el desplazamiento lateral de la solera superior del panel durante cl ensayo.

\section{Probetas}

Los ensayos se harán sobre una muestra mínima de 3 paneles iguales en tamaño, material y procedimiento constructivo.

Antes de efectuar el ensayo se comprobará la rectitud de los paneles y la planeidad de sus caras, como se indica en la norma NCh 806 E Of. 71.

Las probctas tendrán altura, ancho y espesor igual al elemento en uso.

\section{Procedimiento}

a) Apoyar la probeta a lo largo de su base y colocar un tope en el extremo inferior opuesto al costado de aplicación de la carga para evitar desplazamientos horizontales. (Ver Figura $N^{\circ} 3$ ).

b) En el costado de aplicación de la carga, colocar el sistema de anclaje destinado a impedir el levantamiento del extremo inferior. Los tirantes deberán acondicionarse de tal manera que pueda regularse la tensión que es aplicado sobre ellos. Estos tirantes se ajustarán de modo que la tensión no exceda de $9 \mathrm{Kg}$. en cada uno de cllos con anterioridad a la aplicación de las cargas.

c) Leer los tres deformómetros simultáneamente: antes de la aplicación de la carga, cuando la carga está aplicada y cuando la carga se haya retirado.

d) Hacer observaciones parciales en cada estado de carga para detectar las fallas locales, alabeos, desprendimientos, etc.

e) Registrar la carga que produjo la rotura, la carga máxima observada, o la carga que produce una deformación de $10 \mathrm{~cm}$. y anotar las características de la falla.

f) Aplicar las cargas por incrementos aproximadamente iguales con una tolerancia de $\pm 10 \%$ de la carga. Medir la deformación producida por cada incremento de carga. Elegir los incrementos de modo que permitan obtener un número suficiente de puntos para trazar la curva carga-deformación en forma precisa. El número mínimo de incrementos será de cinco y el valor de cada incremento inferior a la quinta parte de la carga supuesta del panel.

g) La velocidad nominal de aplicación de la carga será de $5 \mathrm{~mm} / \mathrm{mín}$., con una tolerancia de $\pm 10 \%$.

h) El ensayo se iniciará con una pequeña carga o sin carga. Si se aplica una pequeña carga inicial, registrar ésta y la deformacion correspondiente. Llevar la carga al primer incremento y anotar la deformación. En seguida llevar la carga a la carga inicial o a cero, según corresponda, y registrar de deformación residual permanente observada. Aumentar la carga en dos incrementos anotándose la deformación y nuevamente volver al estado inicial, registrar la deformación residual observada.

i) Cuando las deformaciones indiquen que el panel se aproxima a su límite de fluencia, abandonar la secuencia descrita en el punto anterior, retirar los instrumentos de medición y llevar la carga hasta la rotura, carga máxima o hasta que el panel se haya deformado $10 \mathrm{~cm}$. 
j) Para cada dial indicador u otro aparato de medición, calcular el desplazamiento como la diferencia entre la lectura cuando la carga está aplicada y la lectura inicial.

k) La deformación residual será la diferencia entre la lectura cuando la carga ha sido retirada y la lectura inicial.

l) Calcular la deformación horizontal y la deformación horizontal residual permanente del panel como la lectura del dial del extremo superior opuesto al costado de aplicación de la carga menos la suma de las lecturas de los otros dos.

\section{Informe}

a) Hacer una descripción de las dimensiones, materiales y características constructivas de los paneles ensayados. En paneles de madera, registrar el contenido de humedad de los elementos estructurales y de los recubrimientos de acuerdo a la norma NCh 176 Of. 53 .

b) Expresar los resultados de los ensayos en gráficos. Las cargas se colocarán, en $\mathbf{N} / \mathbf{m l}$, en las ordenadas y las deformaciones, en mm., en las abcisas.

c) En el gráfico aparecerán las curvas de carga y deformación residual permanente de cada uno de los paneles ensayados.

d) Establecer dos curvas promedio para todo el ensayo, una para la deformación bajo carga y la otra para la deformación residual permanente.

e) Registrar la carga de rotura, la carra a la que se interrumpió el ensayo o la carga que produjc de deformación de $10 \mathrm{~cm}$., indicándose cuál de éstos se consideró.

f) Informar sobre cualquier falla local, alabeos, desprendimientos, etc., observados durante el ensayo y señalar en qué etapa se produjeron.

g) El panel se considerará satisfactorio cuando cumpla con las exigencias de la norma $\mathrm{NCH}$ 806 oF. 71.

\section{MODELACION}

Hasta el momento en nuestro país, los esfuerzos para determinar la curva cargadeformación de un panel sometido a carga horizontal, que en último caso persigue la obtención de las tensiones admisibles y de diseño, están más bien orientados en el examen de paneles con configuraciones específicas. Lo anterior significa que, con la metodología actual, no se busca llegar a un modelo que puede representar cualquier configuración, sino que para cada panel en particular se busca encontrar la tensión de diseño que represente sólo esa configuración.

Si se piensa en el número de variables y combinaciones que intervienen en la construcción de un panel, como son: espaciamiento, tipo y distribución de las fijaciones; dimensiones y módulo de corte de la placa; número de pies derechos; etc., entonces es lógico concluir que la metodología actual es muy limitada.

La norma NCh 1198 E Of 77 "Madera. Construcciones en madera. Cálculo", actualmente en vigencia, utiliza un sistema de evaluación de la capacidad resistente de un diafragma que está basado en lo que se denomina "Panel estándar". Con este sistema, se busca establecer un número mínimo de características comunes para diversos paneles para luego comparar las propiedades resistentes de cada panel con el panel estándar.

Como alternativa de la anterior metodología, se está investigando en el extranjero la posibilidad de representar teóricamente el comportamiento de un diafragma. Existe hasta el 
momento un sin número de modelos propuestos que entregan la función que representa la curva carga-deformación de un diafragma vertical, la mayoría de los cuales son poco prácticos debido a la complejidad de sus términos o a la lentitud de los cálculos que, en muchos casos, obliga el uso de algún sistema computacional.

Sin embargo algunos de estos modelos, se basan en teorías simples, de fácil concepción, y que en definitiva entregan resultados bastante próximos a los reales.

A continuación se entregarán los fundamentos de dos teorías que en la actualidad, son las que tienen mayor aceptación. Ambas consideran que el desplazamiento de un panel sometido a carga horizontal perpendicular a las pies derechos, es la suma de los aportes del corrimiento de las fijaciones y del desplazamiento debido al esfuerzo de corte, despreciando cualquier otro aporte.

La primera de ellas se basa en el equilibrio de las fuerzas que se generan en los clavos al deformarse, producto de la fuerza externa (ver Figura № 4 y № 5).

FIGURA № 4

DEFORMACION DE LAS FIJACIONES

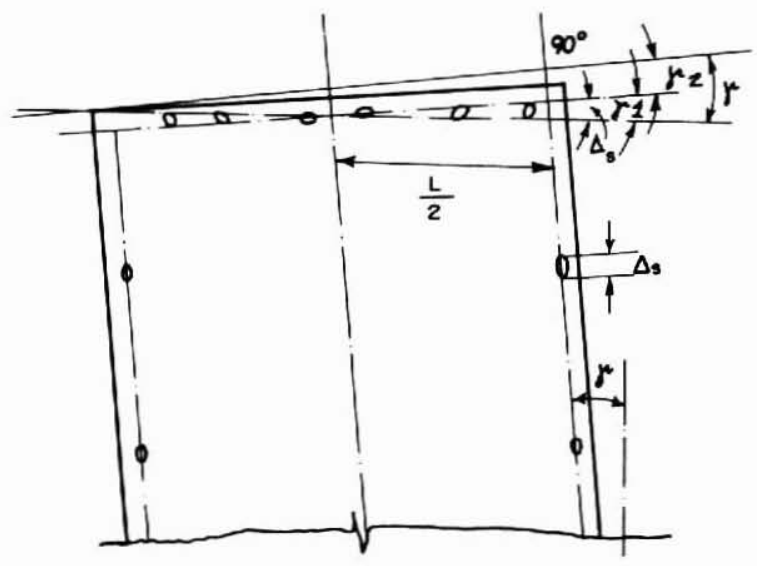




\section{FIGURA $N^{0} 5$}

\section{DIRECCION Y DISTRIBUCION DE LAS FUERZAS}

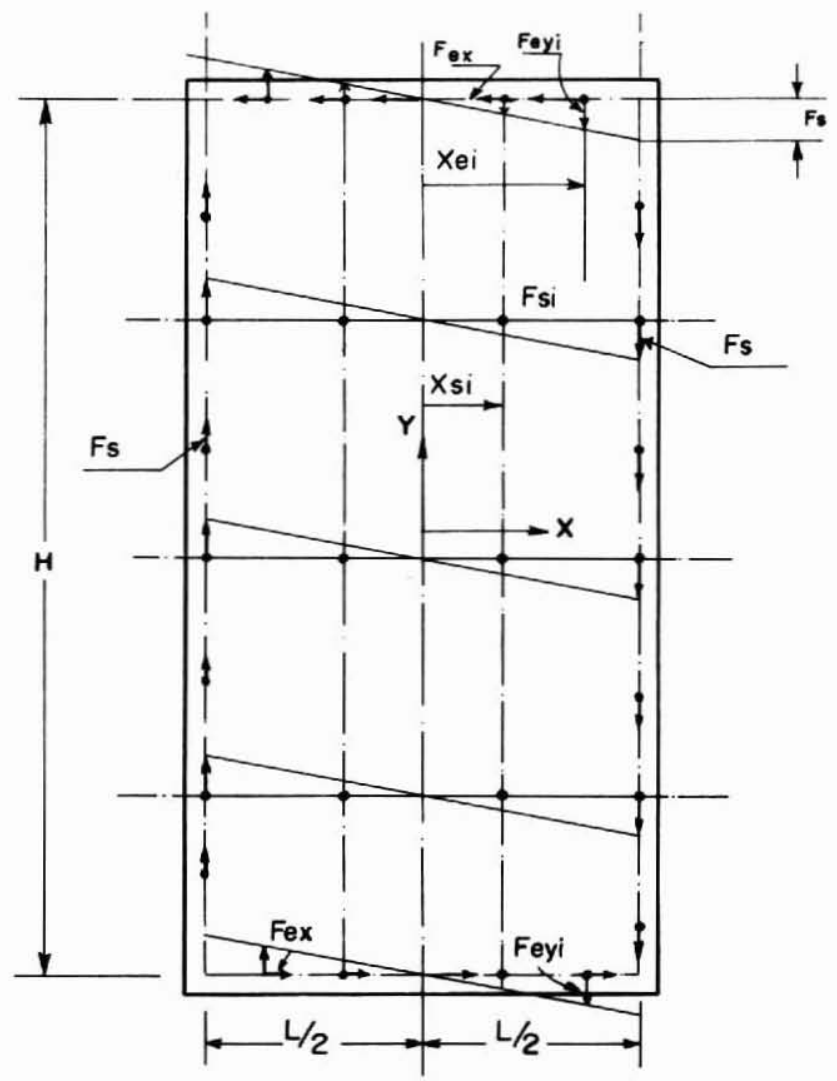

Posteriormente, a la deformación se le adiciona la respectiva desangulación por corte basada en la ley de Hooke para cortadura simple.

La segunda teoría establece un equilibrio, entre la energía externa, debido a la actuación de la fuerza horizontal sobre el panel, y la energía interna, productos de la deformación de los clavos. (Ver Figura №6).

También en este caso, se debe sumar la deformación por corte en la placa. 


\section{FIGURA № 6}

\section{DISTORCION PRODUCIDA ENTRE EL MARCO Y LOS CLAVOS BAJO LA CARGA HORIZONTAL}

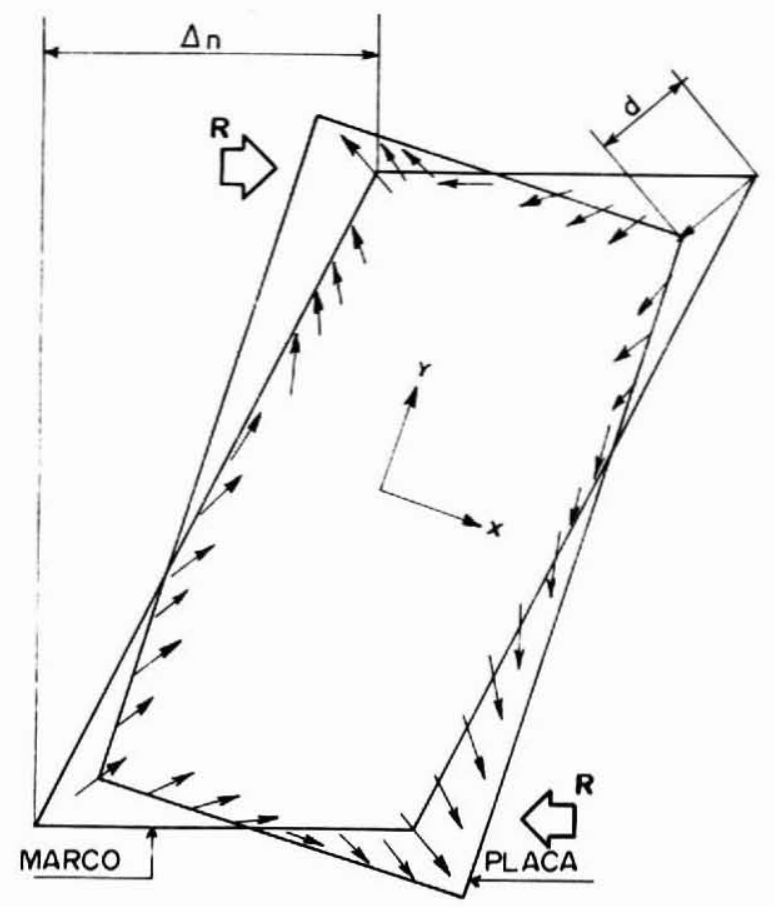

Finalmente, en todos los modelos que se han estudiado, se llegó a la conclusión de que la deformación de los clavos representa sobre el $80 \%$ de la deformación total del panel, y la curva de corrimiento de los clavos tiene la misma forma de la curva carga-deformación del panel.

\section{COMPARACION MODELO-ENSAYO}

Como ya se ha dicho, el corrimiento de las fijaciones constituye el mayor aporte en el desplazamiento del panel, por lo que se hace imprescindible conocer su comportamiento a través de la curva carga-deformación de ellos. 
En general, los modelos que buscan representar la curva carga-deformación del panel, expresan el desempeño de las fijaciones por medio de constantes que se deducen a partir de representaciones no-lineales de curvas de carga-deformación de clavos. Dentro de las diferentes ecuaciones que pueden definir la relación carga-deformación de las fijaciones, existen cuatro formas que son las más aceptadas:

\section{a) Curva Exponencial}

$$
\mathrm{p}=\mathrm{A} \delta^{\underline{B}}
$$

b) Curva Logarítmica

$$
\mathrm{p}=\mathrm{Aln}(1+\mathrm{B} \delta)
$$

c) Curva Asintónica

$$
p=\frac{A+\delta}{B+\delta}
$$

d) Curva Tangente Hiperbólica

$$
\mathrm{p}=\mathrm{A} \tan \mathrm{h}(\mathrm{B} \delta)
$$

En donde:

$$
\begin{array}{lll}
\mathrm{p} & =\text { Carga lateral en la fijación } \\
\delta & =\text { Corrimiento de la fijación } \\
\mathrm{A}, \mathrm{B} & =\text { Constantes. }
\end{array}
$$

Las curvas teóricas a,b,c y d y los rangos de valores se muestran en el gráfico № 1. 


\section{GRAFICO N ${ }^{2} 1$}

\section{CURVAS TEORICAS DE LAS FIJACIONES Y RANGO DE VALORES EXPERIMENTALES}

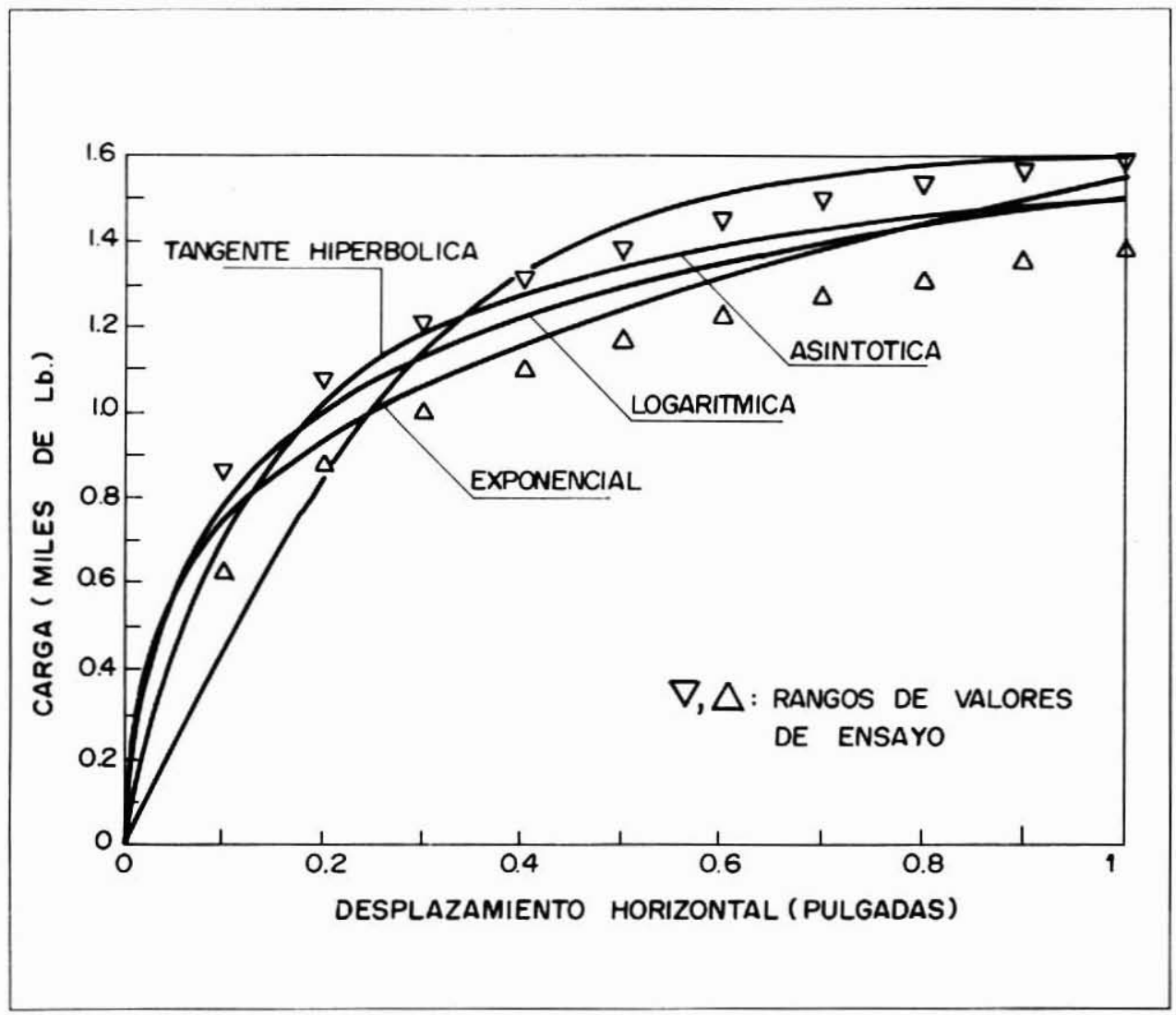
predicción.

De las cuatro curvas anteriores, la forma asintónica proporciona la más exacta

\section{DISEÑO}

Una vez determinada la curva carga-deformación que represente a cualquier muro de corte, se está en condiciones de interpretar sus resultados. Existen distintos criterios para determinar las capacidades admisibles de un panel ante cargas horizontales. Una forma es limitar la distorsión angular del panel.

La norma nacional NCh 1198 E Of 77 establece que la carga admisible para un diafragma, es aquella que induce una deformación de $12,5 \mathrm{~mm}$ en él. Esta deformación, 
representa un criterio poco conservador al aceptar deformaciones de entre piso de 1/192 de altura en muros de $240 \mathrm{~cm}$. de alto. Este alcance, se basa en el efecto que produce dicha distorsión lateral al sobreponer los esfuerzos verticales a que es sometido un muro, generándose a ese nivel de deformación, excentricidades en la acción de las cargas verticales.

Una razón más conservadora para la deformación admisible, sería de $\mathrm{H} / 300(8 \mathrm{~mm}$. en muros de $240 \mathrm{~cm}$. de alto), con lo cual disminuye el efecto de dichas cargas verticales, estando acorde a la normativa extranjera.

\section{CONCLUSIONES}

La importancia de investigar el comportamiento de los muros de corte revestidos con placas, radica en el mejor aprovechamiento generalizado de materiales y mano de obra. Estos muros entregan un aporte rigidizante de gran importancia en la estructura de una edificación, limitando sus deformaciones.

A pesar de los grandes esfuerzos que han desarrollado investigadores extranjeros para determinar algún tipo de formulación sencilla que representa la curva carga-deformación de un panel, esencialmente en base a las propiedades geométricas del mismo, aún no ha sido posible encontrarla, y sólo se ha podido llegar a aproximaciones que, en muchos casos, sólo representan a un limitado grupo de diafragmas, por lo que el tema aún no ha sido agotado, quedando muchos caminos por recorrer.

No obstante lo anterior, hay puntos sobre los cuales existen consenso: el mayor aporte en la deformación de un panel está dado por el corrimiento de las fijaciones. Según esto, las futuras investigaciones deben abocarse, en primer lugar, a desarrollar ecuaciones que representen teóricamente la curva carga-deformación de las fijaciones. En la medida que dichas ecuaciones sean confiables, el modelo que interprete a los paneles será también confiable.

En este artículo se han presentado cuatro tipos de ecuaciones que representan la curva carga-deformación de los clavos. De ellas, la que ha entregado mejores resultados ha sido la forma asintótica.

En lo que respecta a la forma de realizar los ensayos entregada por la norma NCh 802 Of 70 , se puede considerar en términos generales que es adecuada para los requerimientos actuales. Sin embargo, habría que revisar algunos puntos para su optimización. Uno de los más importantes es el que refiere al "límite de fluencia". Este límite es muy subjetivo pues, como se ha visto, la curva carga-deformación de un panel es no lineal y no se aprecian puntos de discontinuidad en ella. Además de lo anterior, entre el límite de fluencia y la carga de rotura no se especifica medir la deformación, lo que implica una zona sin información.

La construcción masiva en base a paneles revestidos con placas rigidizantes, puede tener gran importancia en la economía de nuestro país, debido a su menor costo en comparación con los materiales alternativos como hormigón o acero, lo que contribuiría a una reducción en el déficit habitacional.

En los países desarrollados, la construcción en madera tiene gran importancia y se emplean una gran cantidad de recursos en investigación y fomento de su uso. En nuestro país, la madera sigue siendo considerada como material de segunda categoría en la construcción.

Ya es tiempo de empezar a aprender 


\section{BIBLIOGRAFIA}

1.PATTON-MALLORY, MARCIA; Mc. Cutcheon William J., "Predicting Racking Performance of walls sheathed on both sides", Forest Product Journal, Vol. 37 № 9, Septiembre 1987, pág. 27 a 32.

2. MC. CUTCHEON, WILLIAM J.; "Racking Deformations in Wood Shear Walls", Journal of Structural Engineering, Vol. 110, №2, Febrero 1985, pág. 257 a 269.

3. EASLEY, J. T.; M. Y DODDS, R.H.; Fórmulas for Wood Shear Walls", Journal of Structural Enginnering, Vol 108 № 11, Noviembre 1982, pág. 2460 a 2478.

4. Norma Chilena (NCh 1198 E. Of. 77), "Madera. Construcciones en Madera. Cálculo”, 1977.

5. Norma Chilena (NCh 802 E. Of 70), "Arquitectura y Construcción. Paneles Prefabricados. Ensayo de carga Horizontal”, 1980.

6. Norma Chilena (Nch 806 E Of. 71), "Arquitectura y Construcción. Paneles Prefabricados. Clasificación y Requisitos", 1971.

7. DOWRICK, D.J.; SMITH, P.C.;'Timber Sheather Walls for Wind and Earthquake Resistence", Bulletin of the New Zeland National Society of Earthquake Engineering . Vol 19, № 2, Junio 1986, pág. 15 a 20. 